Revue d'histoire de l'Amérique française

REVUE D.HISTOIRE DE L'AMÉRIQUE FRANÇAISE

\title{
Allégeance et origine : contribution à l'analyse de la crise politique au Bas-Canada
}

\section{Richard LaRue}

Volume 44, numéro 4, printemps 1991

URI : https://id.erudit.org/iderudit/304923ar

DOI : https://doi.org/10.7202/304923ar

Aller au sommaire du numéro

\section{Éditeur(s)}

Institut d'histoire de l'Amérique française

\section{ISSN}

0035-2357 (imprimé)

1492-1383 (numérique)

Découvrir la revue

Citer cet article

LaRue, R. (1991). Allégeance et origine : contribution à l'analyse de la crise politique au Bas-Canada. Revue d'histoire de l'Amérique française, 44(4), 529-548. https://doi.org/10.7202/304923ar
Résumé de l'article

Au Bas-Canada, la crise du système politique implique que les solutions avancées pour faire face aux principaux problèmes dans la colonie remettent en question le régime instauré en 1791. La crise débouche alors sur l'alternative suivante : l'union des Canadas ou l'accès à l'autonomie politique du Bas-Canada. Dans ce contexte, la crise politique se généralise et se transforme en une crise d'identité. L'hypothèse développée ici veut que l'identité politique au Bas-Canada se présente comme un montage symbolique qui unit l'un à l'autre deux modes de la subjectivité politique : l'allégeance et l'origine. Partant de là, la crise d'identité se présente comme la dislocation du montage symbolique assurant l'identité politique bas-canadienne. L’idée d'union est alors légitimée au sein d'une politique de l'origine alors que le programme « autonomiste » repose sur un raisonnement politique entièrement déterminé par l'allégeance. 


\title{
ALLÉGEANCE ET ORIGINE: CONTRIBUTION À L'ANALYSE DE LA CRISE POLITIQUE AU BAS-CANADA
}

\author{
RICHARD LaRUE \\ Département d' histoire \\ Université du Québec à Montréal
}

\section{RÉSUMÉ}

Au Bas-Canada, la crise du système politique implique que les solutions avancées pour faire face aux principaux problèmes dans la colonie remettent en question le régime instauré en 1791. La crise débouche alors sur l'alternative suivante: l'union des Canadas ou l'accès à l'autonomie politique du Bas-Canada. Dans ce contexte, la crise politique se généralise et se transforme en une crise d'identité. L'hypothèse développée ici veut que l'identité politique au Bas-Canada se présente comme un montage symbolique qui unit l'un à l'autre deux modes de la subjectivité politique: l'allégeance et l'origine. Partant de là, la crise d'identité se présente comme la dislocation du montage symbolique assurant l'identité politique bas-canadienne. L'idée d'union est alors légitimée au sein d'une politique de l'origine alors que le programme «autonomiste» repose sur un raisonnement politique entièrement déterminé par l'allégeance.

\begin{abstract}
The crisis of the political system in Lower Canada implies that solutions put forth to confront the main problems of the colony challenge the system of government established in 1791. This crisis leads to the following alternative: the union of the two Canadas or the accession of Lower Canada to a form of self-governement. In this context, the political crisis becomes general and develops into an identity crisis. The hypothesis presented in this paper shows that the political identity of Lower Canada represents a symbolical patchwork which unites two modes of political subjectivity: allegiance and origins. Thus, the identity crisis can be described in terms of the dismantling of the symbolical patchwork ensuring Lower Canadian political identity. The idea of union is then legitimized within a policy of origins, whereas the self-government program rests on a political argument wholly determined by allegiance.
\end{abstract}

Je voudrais proposer ici une lecture de la crise politique au BasCanada portant principalement sur ses aspects structurels. Le problème général que je pose concerne donc la manière dont les conflits politiques coloniaux évoluent vers une crise qui affecte l'ensemble du politique. En d'autres termes, comment passe-t-on d'une crise politique à une crise de la politique dans la colonie? 
L'idée dévelopée dans ce texte est que la crise politique dans la société bas-canadienne devient une crise structurelle à partir du moment où elle se transforme en crise de l'identité politique ${ }^{1}$. Par «identité politique», je désigne ici le montage symbolique par lequel se définit la condition de sujet britannique dans la colonie. Mon hypothèse générale est que, dans le contexte bas-canadien, les individus accèdent à l'existence politique (deviennent des sujets politiques) de deux manières concurrentes: d'abord par un processus de formation de la communauté politique dont rend compte le concept d'allégeance; ensuite par l'assignation aux individus de qualités ou de propriétés spécifiques définissant leur identité comme l'expression d'une origine particulière. Alors que la relation d'allégeance, celle qui s'établit entre un sujet et son souverain, est facilement universalisable, la prise en compte des aspects signifiant l'origine fonctionne comme une spécification au sein de la communauté des sujets britanniques ${ }^{2}$. J'essaierai alors d'établir deux choses. Premièrement, comment allégeance et origine se conjuguent pour produire la figure originale du sujet britannique bas-canadien ${ }^{3}$. Deuxièmement, comment la crise de l'identité politique dans la colonie peut être décrite comme la décomposition du montage symbolique définissant l'identité politique. Cette

1 Selon J. Habermas, une crise générale de la politique doit être analysée comme l'unité de deux crises: une crise des performances du système (crise de régulation) et une crise de l'intégration symbolique de la société (crise de légitimité). Et il ressort clairement des travaux de Habermas, reprenant largement de Max Weber sur cette question, que la légitimité de la domination réfere non seulement à la rationalité du pouvoir, mais aussi à sa faculté de produire l'identité sociale. Dès lors, toute crise générale de la politique est aussi une crise de l'intégration symbolique de la société. J. Habermas, Raison et légitimité. Problèmes de légitimité dans le capitalisme avancé (Paris, Payot, 1978); «Les problèmes de légitimation dans l'État moderne», Après Marx (Paris, Fayard 1985, 1976). Les travaux plus récents de Habermas, surtout sa Théorie de l'agir communicationnel (Paris, Fayard, 1987, 1981), 2 vol., n'apportent rien de radicalement nouveau concernant sa problématique de la légitimité. Sur la théorie des crises de Habermas, Jean-Marc Ferry, Habermas. L'Ethique de la communication (Paris, Preses universitaires de France, 1987), 259-375.

2 De ce point de vue, on peut dire que l'identité fondée sur l'origine est du domaine du semblable, de la reconnaissance de soi dans l'autre. Bref elle se situe du côté de ce que Lucien Sfez analyse comme "égalité de sentiment» dans le modèle que nous aurions hérité des Grecs. Elle vient tempérer l'isonomie, ou égalité «nombrante», qui repose sur l'établissement de la limite par laquelle le groupe produit son unité face au dehors et au dedans de laquelle peut se constituer l'égalité. Voir Lucien Sfez, Leçons sur l'égalité (Paris, Presses de la Fondation nationale des sciences politiques, 1984), 22-35. Toutefois, dans le cadre de l'analyse entreprise ici, l'identité fondée sur la ressemblance fonctionne à l'inverse de cette égalité de sentiment qui vient contrer les inégalités produites par l'isonomie (la multiplication des classes d'inégaux).

3 Au Haut-Canada, le problème peut être posé presque de la même manière. De ce point de vue, je ne ferai qu'en référer à l'étude de David Mills, The Idea of Loyalty in Upper Canada, 1784-1850 (Kingston et Montréal, McGill-Queen's University Press, 1988), 238 p. Toutefois, l'originalité de la problématique bas-canadienne tient au rôle fondamental qu'y joue le problème de la différence d'origine. 
approche porte donc sur la logique des problèmes de légitimité dans la colonie. C'est dire que sans être un pur exercice spéculatif, elle a peu à voir avec une analyse attentive au strict déroulement des événements.

\section{ALLÉGEANCE ET ORIGINE: L'IDENTITÉ POLITIQUE}

La distinction et la séparation de l'allégeance et de l'origine dans les rapports entre les sujets et le souverain britannique avaient été «codifiées» par Sir Edward Coke à l'occasion de son rapport sur l'affaire Calvin ${ }^{4}$. On peut dire que la forme traditionnelle des rapports entre le souverain et ses sujets a alors été problématisée précisément comme l'établissement d'une distinction entre l'origine et l'allégeance. En effet, le nœud de cette affaire est la définition du statut des étrangers au moment de l'accession au trône d'Angleterre de Jacques ler, qui était aussi roi d'Écosse. Aussi essayait-on d'établir que celui qui était né hors de l'allégeance de Jacques $1^{\text {er }}$ roi d'Angleterre était un étranger. De leur côté, les défendeurs, et Coke dans son rapport de l'affaire, essaient d'établir que l'allégeance à la personne de Jacques $1^{\text {er }}$ suffit à fonder l'identité politique du sujet. Pour ce faire, Coke soutient que la qualité de sujet qui découle de l'allégeance, et de l'allégeance seule, ne subit aucune limitation de lieu.

L'allégeance, ainsi que la foi et la fidélité qui sont ses membres et ses parties, sont des qualités de l'esprit et de l'âme de l'homme. Elles ne peuvent être circonscrites dans la catégorie du lieu, car ce serait là confondre les catégories et vouloir amener chose impossible et absurde - la catégorie de la qualité dans la catégorie du lieu. ${ }^{5}$

Par la suite, Coke fait référence à la théorie des deux corps du roi pour se demander à laquelle des deux personnes du roi est due l'allégeance ${ }^{6}$. Or elle est due à la personne physique du roi et non à sa personne politique, dans la mesure où sa capacité politique est une

$4 \quad$ Sir Edward Coke, Reports (Dublin, G. Wilson, ed., 1792-1793), 7 vol. La traduction que j'utilise provient de l'étude réalisée par Richard Marienstras, Le proche et le lointain. Sur Shakespeare, le drame élisabéthain et l'idéologie anglaise aux XVI' et XVII' siècles (Paris, Éditions de Minuit, 1981), chapitre V, «L'affaire Calvin et le statut des étrangers sous Jacques $1^{\text {er }}$ d'Angleterre».

5 Sir Edward Coke cité par Richard Marienstras, op. cit., 159.

6 Signalons que pour Kantorowicz l'affaire Calvin sert à démontrer que le discours des juristes élisabéthains emprunte sa substance au langage théologique en général et à la christologie en particulier qui s'organise autour du problème central des «Deux Natures». Ernst Kantorowicz, Les Deux Corps du Roi. Essai sur la théologie politique au Moyen-Âge (Paris, Gallimard, 1989, 1957), 27ss. 
propriété de sa personne naturelle. Il découle de l'ensemble de cette argumentation que l'allégeance est une relation naturelle entre un sujet et la personne physique du roi. À ce titre, elle est indépendante des formes positives de la $\operatorname{loi}^{7}$. De ce fait il aurait existé en Angleterre une loi naturelle, antérieure à la loi positive et qui unit de naissance les sujets écossais et anglais de Jacques $1^{\text {er }}$, sans égard aux lois différentes les régissant. L'allégeance suffit alors pour définir l'identité politique, en tant que celle-ci se rapporte à une communauté politique naturelle, représentée sur le modèle traditionnel de la communauté familiale et patriarcale. Par la suite, ce sera l'œuvre de la philosophie politique que de montrer comment cette communauté naturelle, organisée selon le modèle patriarcal, ne suffit pas à rendre compte de la nature du gouvernement civil qui, lui, relève de l'artifice ${ }^{8}$. Mais demeurera, avec le contractualisme et l'artificialisme, l'idée que la société civile n'abolit pas la loi naturelle; elle protège en fait les droits naturels des individus, menacés par l'état de guerre de tous contre tous auquel «l'état de nature» tend à s'identifier à la fois comme origine fictive ou comme régression possible.

Le problème majeur que pose une telle conception de la communauté politique dans le contexte colonial vient de son potentiel universalisant qui redouble celui contenu en germe dans l'idée même de domination impériale. On peut dire en effet de cette forme de domination qu'elle «correspond à l'unité des hommes, à l'universalité de la nature, qui veut être reconnue et réfléchie dans et par un pouvoir unique» ${ }^{9}$. Comme pour contrecarrer cet universalisme, et ce de manière explicite après la Révolution française, les Britanniques allaient produire une version de l'identité politique fondée sur le particularisme anglo-saxon ${ }^{10}$. Aux droits de l'homme et du citoyen définis

7 Il faut remarquer ici que le jugement de Lord Mansfield dans Campbell vs Hall, en 1774, fait référence au principe établi par le rapport de Coke sur l'affaire Calvin affirmant que, dans le cas d'une conquête militaire, le roi seul, sans son Parlement, peut modifier les lois du pays conquis tant que celui-ci est régi par ses anciennes lois. C'est-à-dire tant que le roi n'a pas renoncé à sa prérogative et implanté dans ce pays les lois de type britannique. A. Shortt et R. G. Doughty, éd., Documents relatifs à l' histoire constitutionnelle du Canada, 1759-1791, 1: 509.

8 Hobbes sera le premier à faire du gouvernement un produit de l'art humain. Aussi, le deuxième traité sur le gouvernement civil de Locke constitue en fait une réfutation systématique des thèses défendues par Filmer, principal théoricien, à l'époque, de l'idée patriarcale appliquée au gouvernement. J. Locke, Traité du gouvernement civil.

9 Pierre Manent, Histoire intellectuelle du libéralisme. Dix leçons (Paris, CalmannLévy, 1987), 18.

10 Pour une vue d'ensemble du courant réactionnaire en Europe, voir Bertrand Binoche, Critiques des droits de l'homme (Paris, Presses universitaires de France, 1989); aussi la présentation que fait P. Raynaud dans l'édition des Réflexions sur la Révolution de France d'Edmund Burke (Paris, Hachette «Pluriel», 1989), ix-cv. Voir aussi R. Legros, L'idée d'humanité. Introduction à la phénoménologie (Paris, Grasset, 1990). 
en référence à une nature abstraite parce qu'universelle, on opposera les droits du citoyen anglais transmis par héritage. Conception qui trouvera sa forme classique dans l'œuvre de Burke, dont on ne saurait par ailleurs exagérer l'importance pour l'histoire politique canadienne:

...le principe constant de notre constitution a été de faire valoir et d'affirmer nos libertés comme un héritage inaliénable qui nous est venu de nos aïeux et que nous devrons transmettre à notre postérité; comme un bien appartenant en propre au peuple de notre royaume, indépendamment de tout autre droit préalable ou plus large. ${ }^{11}$

Difficile de trouver expression plus directe du refus de l'universalisme abstrait porté par la Révolution française et de sa compréhension des théories du droit naturel. Mais aussi, comment ne pas y voir une solution de rechange à cette autre forme de l'universalisme, celle qui définit la communauté politique sur la seule base de l'allégeance ${ }^{12}$. À l'indifférence affichée par la théorie de l'allégeance pour les régimes politiques existants, la problématisation burkienne de l'identité oppose une conception de la subjectivité politique qui n'existe que dans et par le droit positif et particulier. Ce que l'allégeance pouvait universaliser, la forme patrimoniale de la subjectivité politique l'associe à une sorte de particularisme généralisé qui appelle l'idée de race ${ }^{13}$. Comme le souligne Reginald Horsman à la suite des travaux de L. P. Curtis, la croyance en un âge d'or anglo-saxon d'où viendraient les libertés britanniques, autrefois utilisée pour défendre les droits du peuple, s'est transformée au $\mathrm{XIX}^{e}$ siècle en une justification de la domination impériale sur les autres peuples ${ }^{14}$. Cette façon de fonder l'identité politique dans le particularisme anglais sera donc une des composan-

11 Burke, op. cit., 42.

12 Toutefois, la critique empiriste de l'universalisme abstrait se situe du côté de la modemité. À cet égard, il n'est pas inutile de signaler ici que la critique que fait Burke de l'abstraction ne réfêre pas à un passé médiéval qu'il s'agirait de faire revivre, mais plutôt à «l'expérience», en tant que celle-ci est une série événementielle régionale et contingente. B. Binoche, op. cit., 12. Vincent Descombes rappelle aussi fort à propos que «la seule philosophie qui pense jusqu'au bout l'opposition de la raison individuelle et de tout ce qui serait reçu, transmis, traduit, donné par une autorité extérieure est l'empirisme». Philosophie par gros temps (Paris, Les Éditions de Minuit, 1989), 150.

13 «La notion d'héritage, appliquée à la nature même de la liberté, a été le fondement idéologique qui conféra au nationalisme anglais cette curieuse touche de sentiment racial à partir de la Révolution française». H. Arendt, Les origines du totalitarisme. $2^{c}$ partie: L'impérialisme (Paris, Seuil, coll. «Points», 1984, 1951), 98.

14 Reginald Horsman, «Origins of Racial Anglo-Saxonism in Great Britain Before 1850», Journal of the History of Ideas, 37,3 (July-September 1976): 387; L. P. Curtis, AngloSaxons and Celts: a Study of Anti-Irish Prejudice in Victorian England (Bridgeport, Conn., 1968). 
tes nécessaires du développement de l'empire britannique ${ }^{15}$. À plus forte raison lorsque celui-ci sera produit idéologiquement et politiquement comme l'accomplissement d'une destinée visant à répandre partout sur la planète les formes de la civilisation britannique ${ }^{16}$. Dans ce contexte, la notion de «civilisation britannique» telle qu'elle est utilisée dans la polémique coloniale désigne empiriquement le contenu de la notion d'origine. Dans le cadre de cette analyse, et en laissant provisoirement de côté l'aspect théorique de cette question, «origine» et «civilisation» valent l'une pour l'autre.

Schématiquement, on pourrait dire que les hésitations de la politique impériale après la conquête de la Nouvelle-France, les avances et reculs en ce qui concerne la «britannisation» de la société coloniale témoignent du caractère contingent de l'articulation de l'allégeance et de l'origine en tant que forme de l'identité politique ${ }^{17}$. Le cadrage de ces deux éléments, la force de l'un par rapport à l'autre, ne répond pas uniquement, ni même principalement, d'une logique juridique quelconque $^{18}$. Et c'est la raison pour laquelle le problème posé ici ne peut se réduire à la question du rattachement juridique de la colonie à la métropole ${ }^{19}$. Plus précisément, la politique impériale est essentiellement une «politique de la population». C'est la faible immigration britannique vers la colonie conquise qui entraînera l'abandon de la politique de «britannisation» définie par la Proclamation royale de 1763. De même que l'afflux massif des Loyalistes américains après 1783 sera l'élément qui motivera la métropole à finalement concéder à sa colonie une constitution similaire à celle de la Grande-Bretagne. Mais quoi qu'il en soit des motivations de la politique impériale et de

15 Ainsi, dans une optique différente de celle exposée ici, I. Wallerstein présente-t-il la race comme une catégorie exprimant l'identité politique du point de vue de la division axiale du travail dans l'économie-monde. C'est-à-dire que la race apparaît comme une forme de l'identité liée à l'opposition entre le centre et la périphérie. I. Wallerstein, "La construction des peuples: racisme, nationalisme, ethnicité», Wallerstein et Balibar, Race, nation, classe. Les identités ambiguës (Paris, La Découverte, 1988), 107.

16 Sur l'évolution de l'idée impériale en Grande-Bretagne, P. Ward, Colonial SelfGovernment: the British Experience 1759-1856 (Londres, MacMillan, 1976), 211 ss.

17 Comme le dit P. Lawson, «The pertinent questions about bringing this alien society under imperial rule, hinted at by the pamphleteers in 1760-61, had never really been addressed by any leading politicians. No one stopped to think of the magnitude of the task facing the government..." The Imperial Challenge. Quebec and Britain in the Age of the American Revolution (Montréal et Kingston, McGill-Queen's University Press, 1990), 24.

18 L'idée même d'un «droit de Conquête» qui ne soit pas seulement le droit du plus fort est incertaine. En outre, comme le fait remarquer P. Lawson, on peut penser que c'est l'expérience acquise dans la «Province of Quebec», telle que l'analyse Wedderburn dans son rapport de 1772, qui aurait servi de fondement au raisonnement tenu dans le célèbre jugement de Lord Mansfield. P. Lawson, op. cit., 121.

19 Sur cette question, J.-E. Côté, «The Reception of English Law», Alberta Law Review, 15 (1977): 29-92. 
son cadre géostratégique, à partir de 1791 l'identité politique au BasCanada se présente comme un cadrage de l'allégeance et de l'origine. D'un côté, la qualité de sujet britannique s'exprime par l'exercice de droits politiques assimilables, mais non identiques à ceux que garantissent les pratiques constitutionnelles anglaises; de l'autre, les droits reconnus à la majorité canadienne par l'Acte de Québec sont maintenus. Il devient donc possible d'être un loyal sujet de Sa Majesté et d'être un francophone catholique qui administre ses affaires en suivant les préceptes de l'ancien droit canadien, tout en participant à la vie politique au même titre que les colons d'origine britannique. En outre, depuis l'Acte de Québec, les tenures française et anglaise coexistent légalement dans la colonie. Situation d'ensemble qui comportera toujours une large part d'incertitude et d'indétermination, notamment en ce qui concerne l'organisation du droit privé ${ }^{20}$. Mais globalement, on peut dire que la tension inscrite au cœur même de la forme de l'identité politique bas-canadienne sera à la fois le moyen de la gestion pacifique de la colonie et la possibilité formelle de la crise politique.

\section{L'IDENTITÉ DU SEUL POINT DE VUE DE L'ORIGINE: L'IDÉE D'UNION}

La crise de la politique se présentera dans la colonie comme une tentative de recomposer l'identité politique sur la base d'un seul des deux aspects mis en relation au sein de la figure symbolique du sujet politique bas-canadien. Procédure que l'on peut décrire comme l'accès à l'autonomie et à la dominance de l'un des pôles de l'identité politique au détriment de l'autre. C'est ainsi du moins que se présente l'idée de réunir les colonies constituées en 1791: comme la revendication d'une identité politique qui reposerait exclusivement sur l'origine ${ }^{21}$.

20 Voir E. Kolish, «The Impact of the Change in Legal Metropolis on the Development of Lower Canada's Legal System: Judicial Chaos and Legislative Paralysis in the Civil Law, 1791-1838», Canadian Journal of Law and Society/Revue canadienne de droit et société, 3 (1988): 1-26; J. E. C. Brierley, "The Co-Existence of Legal Systems in Quebec: Free and Common Soccage in Canada's Pays de droit civil», Les Cahiers de Droit, 20,1-2 (1979): 277. 288.

21 Dans la mesure où l'on concentre l'attention exclusivement sur la question de l'identité politique, l'approche développée ici se distingue radicalement de celle de P. J. Smith sans la remettre en question. Selon cette analyse, trois thèmes se dégagent des propositions d'union mises de l'avant par les Tories avant la Confédération: le renforcement de la prérogative royale dans les colonies d'Amérique du Nord, la création de débouchés pour l'ambition légitime et l'importance du développement économique. Peter J. Smith, «The Ideological Origins of Canadian Confederation", Canadian Journal of Political Science/Revue canadienne de science politique, 20,1 (mars 1987): 3-29; "The Dream of Political Union: Loyalism, Toryism and the Federal Idea in Pre-Confederation Canada», Ged Martin, ed., The Causes of Canadian Confederation (Fredericton, Acadiensis Press, 1990), 148-172. 
On peut commencer par étudier brièvement la crise politique déclenchée par le projet d'Union de 1822. Cet épisode de l'histoire coloniale nous intéresse ici non pas comme un événement qu'il faudrait reconstituer, mais plutôt comme un moment où s'affrontent des discours justifiant une forme ou l'autre de l'État colonial. Les arguments et justifications amenés en faveur de chacune des positions définies par cette tentative révèlent que le cadrage de l'identité politique coloniale opéré en 1791 constitue l'un des principaux enjeux de la manœuvre alors tentée. Ainsi, une pétition d'habitants d'origine britannique de la province du Bas-Canada en faveur du projet d'Union pose-t-elle de manière explicite le problème de la distinction entre l'origine et l'allégeance ${ }^{22}$. On y affirme ainsi que le Bas-Canada est séparé en deux parties: la zone seigneuriale ou «Bas-Canada français» où sont quand même établis près de quarante mille habitants d'origine britannique et les townships peuplés exclusivement «d'habitants de naissance et d'extraction britannique, et de loyalistes américains». Les éléments que l'on énumère pour décrire la différence entre les deux parties sont: la langue, la tenure et la religion. La définition des différences d'origine appelle donc une autre distinction, celle du privé et du public. Pour le dire sans détour, l'origine semble recouvrir les contours du domaine privé (la tenure et les lois, la religion, la langue) dès lors que le droit public, en fait l'allégeance, ne suffit plus à assurer l'identité politique. Toutefois, l'inachèvement de la séparation du public et du privé - inachèvement dû à la fois au caractère idéologique de cette séparation et à l'état des rapports sociaux - empêche de réduire notre problème à la seule question de la réception des lois anglaises et du maintien du droit civil français dans la colonie. Par exemple la religion est, au départ, une question de caractère public. Elle est une des conditions de l'exercice des droits politiques dans le régime britannique. Plus tard elle devient une affaire purement intime, interne à la sphère privée. Elle sera alors pensée comme une composante des lois et coutumes.

Dans ce contexte, la proposition d'union des deux provinces sépare les deux termes de l'identité politique. Premièrement, elle fait de l'allégeance l'unique composante de l'identité politique mise en place avec l'Acte constitutionnel de 1791. Deuxièmement, elle prétend reconstruire cette identité sur la seule base de l'origine, identifiée au domaine privé. La suite de la pétition montre bien comment origine et domaine privé en viennent à valoir l'un pour l'autre. Ainsi le maintien d'un espace politique où existe et se développe un régime

22 R. G. Doughty et N. Story, éd., Documents relatifs à l'histoire constitutionnelle du Canada, 1819-1828, 3: 133. 
d'origine française est présenté comme le développement d'une nation étrangère au sein de l'empire. Ou encore, le maintien du Bas-Canada seigneurial et français est perçu comme la préservation d'un système «calculé à détourner les personnes d'origine britannique et leurs descendans de s'établir sur les terres en friche de la Couronne» ${ }^{23}$.

Et comme il s'agit de recomposer l'identité politique, le problème de l'origine pose directement celui de l'allégeance. En clair: peut-on réellement compter sur la loyauté d'une nation étrangère au sein de l'empire?

...un des objets les plus grands et les plus glorieux des nations qui élèvent et protègent des colonies, doit être d'établir une race qui perpétue dans les siècles à venir la ressemblance honorée de la mère-patrie; et ils ont senti qu'il ne pouvait être d'accord avec la dignité ni avec les intérêts de la Grande-Bretagne, pour en faire par la suite une image de la France sous le rapport de la langue et des lois lorsque la France est exempte de tous les frais. ${ }^{24}$

Dans l'optique où l'origine est la seule norme en fonction de laquelle on veut définir l'identité politique, la ressemblance existant du fait de la communauté d'origine devient l'unique garant de la loyauté coloniale $e^{25}$. Elle problématise le rapport de la société coloniale à la société britannique sous le registre de l'analogie, donc de la hiérarchie. En maintenant la production de l'identité politique dans le cadre de la ressemblance, de la communauté d'origine, on évite de penser les rapports entre la colonie et la métropole comme un rapport égalitaire. Ce faisant, on inclut la production de l'identité politique coloniale dans la vision traditionnelle, organique, de l'empire britannique.

Cette ressemblance s'organise alors non seulement au sein des institutions de la sphère privée, mais encore tend-elle à recouvrir le domaine de l'intime, tant il est vrai qu'on l'on ne vise rien de moins que l'assimilation graduelle par «les habitants de toute origine des sentiments britanniques» ${ }^{26}$. L'idée de race est alors associée au droit privé ou à la tenure pour prendre en charge la définition de la ressem-

23 Ibid., 134. On ajoute aussi que près de 100000 émigrés d'origine britannique n'ont fait que passer au Bas-Canada pour aller s'établir aux États-Unis où ils se sentent moins étrangers que dans cette colonie britannique.

24 Ibid., 135.

25 Ici, la notion de ressemblance ne désigne que le seul établissement d'un rapport fondé sur la communauté d'origine. Elle est différente de la notion de similitude par laquelle Tocqueville définit l'égalité de condition. A. de Tocqueville, La démocratie en Amérique (Paris, Flammarion, 1981), 2: 22ss.

26 R. G. Doughty et N. Story, op. cit., 135. 
blance avec la mère-patrie. Du coup, la condition de sujet britannique dans la colonie réfère non plus au domaine public (la problématique de l'allégeance) mais se pense au sein de la problématique de la race, ou de la «civilisation». C'est ce qui explique que les problèmes exposés par les pétitionnaires soient au-delà de revendications concernant le réaménagement de la représentation au sein des colonies. Il faut plutôt transformer la nature même des sujets de Sa Majesté. Comme le souligne encore une «Pétition des Habitans de Québec»:

L'expérience de trente années a maintenant démontré les vices de l'acte du Parlement britannique de la 31 Geo. 3 c. 31 , qui divisait la ci-devant province de Québec pour en former les provinces du Haut et du Bas-Canada. C'est à cette division que vos pétitionnaires attribuent l'état de l'inefficacité actuelle de leur législature, et le défaut de mesures nécessaires pour pénétrer la population entière du pays de sentiments convenables au caractère de sujets britanniques... ${ }^{27}$

En fait, on affirme que le cadrage de l'identité politique effectué en 1791, qui pose l'irréductibilité de l'allégeance à l'origine, empêche la colonie d'acquérir un statut véritablement britannique dont la vérité serait de l'ordre du sentiment.

Le projet d'Union de 1822 implique une vision de l'identité politique reposant sur le domaine de l'intime plutôt que sur des règles ou des coutumes relevant du droit public. Alors la communauté fondée sur l'origine (la ressemblance) peut prendre la place de l'allégeance dans la production de l'identité politique. On retrouvera plus tard le même raisonnement dans le rapport Durham. Ce rapport est l'un des textes où se révèle le plus clairement la forme même que prend la crise de l'identité politique lorsque le pôle de l'origine l'emporte sur l'allégeance. L'argumentation du rapport Durham est trop touffue et par trop connue pour qu'il soit question de la reprendre ici dans son entier. Je me contenterai d'indiquer ce en quoi il peut contribuer à la démonstration entreprise ici.

La valorisation de l'origine apparaît d'abord dans l'analyse que fait Durham des causes de la rébellion. On peut décrire sa présentation des causes de la crise du système politique dans les colonies comme une tentative d'établir qu'elle résulte d'une régression vers un état «pré-démocratique» où la représentation est détournée de son fonctionnement normal, reposant sur le système de contrepoids et de limitation des pouvoirs, au profit de la polarisation des instances représentatives. Cette régression prend deux formes. D'abord, au Bas-

27 Ibid., 123. 
Canada, elle se présente comme l'unification du peuple sous le seul rapport de l'origine ou de la race. Ce qui se traduit par le dysfonctionnement des institutions de la sphère publique et de la société civile (la presse, les partis politiques, les jurys). On n'a pas à rappeler ici les exemples cités par Durham témoignant de l'absence de communication dans la vie civile entre les deux races. Disons simplement que pour lui, là où le commerce et la raison auraient dû provoquer la diversité et la discussion, on ne trouve que l'exclusion et la fausse unification, c'est-à-dire le rassemblement sur la seule base raciale ${ }^{28}$. Ainsi la société civile est-elle totalement déterminée par l'affrontement des races alors que le fonctionnement de la sphère publique est détourné par l'abus des considérations émergeant de l'intime, de l'identitaire. Ce qui permet cette régression, c'est l'unité contre nature, anti-démocratique - car fondée sur l'illettrisme, l'ignorance et la naïveté - du peuple français du Bas-Canada. On peut alors remarquer que Durham reproche surtout aux Canadiens d'origine française de surmonter de manière «illégitime» la séparation entre représentants et représentés qui fonde le système de la représentation démocratique tel qu'on le concevait à l'époque. Et, dans la plus pure optique tocquevillienne, il en attribue la cause à l'égalité de condition qui caractérise les sociétés américaines ${ }^{29}$. On sait que pour Tocqueville, l'égalité de condition telle qu'elle est donnée dans le Nouveau Monde pousse l'individualisme à dégénérer en atomisme. C'est-à-dire que la valorisation de l'usage individuel de la raison au détriment des médiations traditionnelles tend à se transformer en privatisme généralisé. Dès lors on n'a plus affaire à des individus libres et égaux, mais à des atomes agglutinés les uns aux autres, ayant sacrifié leur indépendance au profit de l'opinion commune et, finalement, au profit d'un pouvoir qui, du coup, n'a plus de limites. L'égalisation des conditions peut ainsi rendre compte à la fois de l'existence et de la disparition de l'autonomie individuelle ${ }^{30}$. Or, pour Durham, tout se passe comme si les Français d'Amérique en étaient arrivés à ce stade - «atomisme» et agglutination - sans avoir préalablement passé par le développement de l'autonomie subjective, soumis qu'ils ont été à l'absolutisme le plus rétrograde. C'est pourquoi Durham oppose l'ignorance et la confiance

28 Par cette expression «fausse unification» je désigne l'existence de l'unité a priori, de type absolutiste, d'une partie de la population, indépendamment des règles devant présider à la vie politique «démocratique» et moderne et dont le modèle est celui de la sphère publique bourgeoise, tel que l'a analysé Habermas dans L'espace public. Archéologie de la publicité comme dimension constitutive de la société bourgeoise (Paris, Payot, 1978).

29 C. P. Lucas, ed., Lord Durham's Report on the Affairs of British North America (Oxford, 1970, 1912), vol. 2. (dorénavant, Report), 32ss.

30 Tocqueville, op. cit., 434 ss. 
aveugle des Français envers leurs représentants à l'indépendance d'esprit des colons anglais. Du côté du Haut-Canada, Durham trouve aussi un phénomène analogue dont témoigne le nom même, sinon la chose, de «Family Compact». Ici, ce qui est en cause c'est l'absolutisme, ou «irresponsabilité», de la clique au pouvoir qui gère la chose publique en fonction d'intérêts privés et de relations personnelles. On pourrait alors penser que la question de l'origine n'est pas à ce point déterminante dans la pensée de Durham puisqu'il rend compte des problèmes du Haut-Canada sans y faire référence. Ce serait oublier que pour lui l'explication de la violence du conflit réside précisément dans une différence d'origine: celle existant entre les Canadiens anglais nés au Canada ou en Amérique et ceux qui sont nés en Angleterre. Les premiers désirent ardemment que les natifs de la colonie se réservent les fruits du patronage, alors que les seconds sont plus enclins à resserrer le lien avec la métropole ${ }^{31}$. Est-ce aller trop loin que d'en conclure que la catégorie d'origine a une portée générale dans la pensée politique de Lord Durham et qu'elle n'apparaît pas de manière simplement épisodique en raison de la nature exceptionnelle, surprenante, du conflit politique au Bas-Canada?

Non seulement l'analyse des causes de la crise, mais encore les solutions proposées par Durham sont entièrement déterminées par la volonté de penser les rapports politiques sur la seule base de l'origine. La solution de Durham peut être décrite comme un recadrage de l'identité politique où la notion de race englobe celle d'allégeance. Il s'agit simplement d'annuler la protection que lui accordait le cadrage réalisé en $1791^{32}$. On postule alors que le cours normal des choses, c'est-à-dire la mise en place d'un système politique où dominent les Britanniques, portera les individus français à abandonner «leurs vains espoirs de nationalité». À s'en tenir au seul plan de ce calcul qui attribue aux Français la même capacité qu'aux citoyens d'origine britannique, on peut bien faire de Durham un libéral bon teint pour lequel le racisme est une chose ignoble ${ }^{33}$. Mais cela n'empêche que pour Durham la nature et la forme du régime politique sont déterminées par des qualités possédées par les individus du fait de leur

31 Report, 154.

32 Voir la critique que fait Durham de la politique mise en œuvre par la métropole avec l'Acte constitutionnel de 1791. Report, 63ss.

33 Ainsi Durham affirme-t-il que, si le conflit politique au Bas-Canada s'est présenté comme l'opposition des conservateurs et des radicaux ou républicains, c'est parce que la seule notion de querelle raciale apparaît si révoltante dans le monde civilisé qu'elle doit habiller les protagonistes d'autres noms que ceux qui les désigneraient justement, à savoir ceux qui réferent à leur identité raciale. Report, 15 . 
origine $^{34}$. L'analyse comparative que fait Durham des qualités des deux peuples en présence ne vise qu'à établir cela. Les Français, en tant qu'ils sont des Français «d'ancien régime», sont inaptes à la démocratie de type anglais. Alors que les citoyens coloniaux d'origine britannique ont en eux les qualités de volonté et d'entreprise, ainsi que l'indépendance d'esprit nécessaires au bon fonctionnement d'un gouvernement constitutionnel. Ce serait un non-sens de croire que les citoyens d'origine française, du seul fait de leur allégeance à $\mathrm{Sa}$ Majesté, pourront aussi acquérir ces qualités, tant que la forme du régime protégera leur identité nationale distincte. Autrement dit, le rapport Durham repose tout entier sur l'idée qu'il ne suffit pas d'être né dans l'allégeance de Sa Majesté pour être un bon sujet anglais, mais encore faut-il posséder des qualités qui ne se transmettent qu'au sein de la civilisation britannique, par héritage et expérience. Et à ce propos, son argumentation présentant les institutions municipales comme lieux d'apprentissage de la démocratie et du gouvernement est éloquente.

Mais alors, en proposant un recadrage de l'identité où la qualité de sujet est soumise à la catégorie de l'origine, Durham ouvre la possibilité de penser la ressemblance comme une égalité. C'est exactement ce qu'il met en jeu lorsqu'il propose de concéder la responsabilité ministérielle aux colonies. En effet, les mêmes causes (la race ou l'origine anglaise) produisant les mêmes effets, il est impératif de procurer aux colonies une forme de gouvernement qui ne les fasse pas regarder le voisin américain avec envie. Un passage du rapport illustre bien ce glissement théorique de la ressemblance vers l'égalité:

Nulle société importante d'hommes libres et intelligents ne sera longtemps satisfaite d'un système politique qui place ces gens, en même temps que leur pays, dans un état d'infériorité vis-à-vis de leur voisin. ...Tandis que son voisin d'outre-frontière se gourme d'importance à l'idée que son vote exerce quelque influence sur les conseils et que lui-même participe au progrès d'une nation puissante, le colon [britannique] sent l'influence étouffante de la société étroite et subordonnée à laquelle il appartient. ${ }^{35}$

34 Ce pourquoi on ne peut souscrire totalement au plaidoyer en faveur d'un Durham uniquement libéral, pas plus raciste qu'un autre. Ou alors il faut dire qu'en tant que penseur libéral, il est aussi raciste que les autres. De la même manière on ne peut réduire la polémique politique dans les colonies à la seule opposition de «l'humanisme civique» et de «l'idéologie commerciale». L'idée d'union des deux provinces indexe nécessairement la forme du régime politique à des qualités collectives (ethniques) portées par les individus.

35 Report, 310-311. Traduction extraite de l'édition partielle publiée par Denis Bertrand et Albert Desbiens, Le Rapport Durham (Montréal, L'Hexagone 1990, 1969), 255. 
On notera que le sentiment d'infériorité éventuellement ressenti par les sujets coloniaux provient de ce qu'ils possèdent des qualités (la liberté et l'intelligence) qui les rendent semblables à leurs voisins, alors que la situation politique leur fait faire constamment l'expérience de leur infériorité. C'est en ce sens que la ressemblance peut fonder l'égalité, voire l'indépendance. Alors que bien évidemment, ce danger ne se présente pas en ce qui concerne les sujets d'origine française du Canada $^{36}$. Dans cette optique, les propositions faites par Durham concernant la responsabilité ministérielle - tempérée il est vrai par une définition stricte des compétences impériales - sont des conséquences directes de sa conception de l'identité politique. Celle-ci se présente alors comme un nouveau cadrage où ce sont les qualités liées à l'origine des sujets qui représentent l'inscription du régime politique dans la nature des choses et non pas la relation d'allégeance entre les sujets et le souverain ${ }^{37}$.

\section{L'ALLÉGEANCE ET LA CRISE DE L'IDENTITÉ POLITIQUE}

Symétriquement à ce discours, s'en développe un autre qui arrive à la dissolution critique de l'identité politique coloniale en ne considérant que l'allégeance dans la définition de cette identité. Ce discours apparaît d'abord simplement comme la défense de la constitution de 1791 devant le projet d'Union de 1822 . On ne fait alors que proclamer la prédominance de l'allégeance sur l'origine.

Ils [les Canadiens d'origine française] ne sont pas des étrangers dans ce pays où ils sont nés; ils réclament, en leur qualité de sujets britanniques, les mêmes droits que ceux de tous les autres sujets de Sa Majesté dans ces colonies. Voilà les droits que leur confère leur naissance. Et cependant, les partisans du projet de loi sur l'union espéraient les priver de ces droits dont la jouissance leur fut solennellement assurée par l'acte de la $31^{\mathrm{e}}$ de Sa feue Majesté, à la suite des discussions circonstanciées qu'il suscita

36 Parmi les nombreux étonnements de Durham, il y a celui qu'on ait pu trouver une ressemblance quelconque entre les Français du Bas-Canada (population singulièrement inerte et ignare) et les Américains, dont le système démocratique témoigne de l'indépendance et de l'élévation d'esprit. Report, 26. Ailleurs dans le rapport, la dissemblance entre les deux peuples est présentée comme un des arguments assurant l'improbabilité de la diffusion des idées républicaines dans le Bas-Canada et d'une alliance entre celui-ci et les États-Unis. Report, 265.

37 Cela, bien entendu, ne suffit pas à faire du rapport Durham un projet de dissolution de l'empire. Mais comme le fait justement remarquer J. Azjenstat, la seule solution de remplacement que propose Durham à la force armée pour maintenir l'intégrité de l'empire, c'est l'intérêt ou le désir qu'auraient les coloniaux de faire partie de l'empire, une fois celui-ci réformé. J. Azjenstat, The Political Thought of Lord Durham (Toronto, University of Toronto Press, 1988), 47. 
alors en conformité des idées justes et libérales des politiques les plus éclairées du temps. ${ }^{38}$

Il s'agit alors simplement de faire respecter les droits que garantit la relation d'allégeance, tels que codifiés par les actes de 1774 et de 1791. Or, très rapidement, et en fonction d'événements politiques dont il est inutile de reprendre ici la description, cette problématisation de l'identité politique va déboucher sur une opération visant à «absolutiser» le pôle de l'allégeance dans la formation de l'identité politique $^{39}$. On peut suivre le développement de cette crise à travers deux épisodes qui ont traduit dans les faits la volonté du parti patriote de porter la lutte politique sur le plan de la légitimité et de l'identité politique: l'adoption des 92 Résolutions et le mouvement des assemblées publiques.

Les 92 Résolutions furent rédigées comme un réquisitoire pouvant justifier la rupture avec la métropole. Je propose de considérer cette rupture non plus seulement sous l'angle de l'affirmation de l'identité nationale des Canadiens contre l'empire britannique, mais aussi comme l'éclatement de l'identité politique existant depuis 1791. Et l'on peut même penser que, dans la crise de la politique bas-canadienne, cet éclatement précède l'affirmation de l'identité nationale. Allégeance et origine, au lieu de coexister et d'interagir l'une sur l'autre comme dans le modèle de 1791, en viennent à s'opposer de manière frontale, au point de se détruire mutuellement, chacune d'elles prétendant fournir un principe d'identité politique indépendant de l'autre.

C'est toutefois la valorisation exclusive de l'origine qui apparaît d'abord dans le texte des 92 Résolutions. Mais de manière négative, c'est-à-dire comme la stratégie de l'adversaire. Toute la critique du rôle de l'exécutif et du Conseil législatif, ainsi que de la politique britannique face à la colonie, apparaît comme la dénonciation d'un traitement injustement fondé sur la distinction des origines. En traitant de manière injuste les sujets britanniques d'origine française, le gouvernement impérial aurait ainsi violé leurs droits de sujets britanniques, ceux qui sont définis par leur allégeance à la Couronne britannique. Alors qu'eux sont restés loyaux «même dans les circonstances les plus difficiles, sous des Administrations Provinciales qui foulaient

\footnotetext{
147.

38 «Lettre de Papineau à Wilmot, 16 février 1822^, A. G. Doughty et N. Story, op. cit.,

39 Ce processus a souvent été décrit comme l'abandon par les Patriotes de leur «britannisme», à la fois comme sentiment et comme stratégie, au profit du républicanisme américain. Voir Lionel Groulx, «Le britannisme des Patriotes», Revue d'histoire de l'Amérique française, 5,3 (décembre 1951): 416-425.
} 
aux pieds les droits et les sentiments les plus chers à des Sujets Britanniques ${ }^{40}$. L'objectif est déclaré dès l'ouverture du texte: montrer que la politique britannique dans la mesure où elle n'opère qu'en fonction de l'origine annule l'allégeance. Ainsi, l'identité politique fondée sur l'articulation de l'origine et de l'allégeance entre en crise. De ce point de vue, les résolutions 47 et 48 sont certes centrales:

47. Résolu, ...Que la fidélité des Peuples et la protection des gouvernements sont des obligations corrélatives, dont l'une ne saurait longtemps exister sans l'autre; que par suite des défectuosités qui se trouvent dans les lois et constitutions de cette Province, et de la manière dont ces lois et constitutions ont été administrées, le Peuple de cette Province n'est pas suffisamment protégé dans sa vie, ses biens et son honneur; et que la longue suite d'actes d'injustice et d'oppression dont il a à se plaindre, s'est accrue en violence et en nombre avec une rapidité alarmante pour la présente Administration. ${ }^{41}$

Il s'agit alors de poser l'allégeance comme un contrat dont les termes n'auraient pas été respectés par la métropole, ce qui délivre le «Peuple de cette Province» de ses obligations envers le souverain. De cette façon, c'est la forme de l'identité politique qui entre en crise, dans la mesure où les droits qui auraient été violés sont ceux-là mêmes qui «furent assurés aux sujets de Sa Majesté sur la foi et l'honneur national Anglais et sur celle des Capitulation et Traités» ${ }^{42}$. La figure symbolique du sujet britannique dans les colonies se désagrège, devient impossible. Ce qui auparavant opérait la mise en correspondance des deux composantes de l'identité politique devient alors un principe de dispersion. Origine et allégeance, loin de s'impliquer l'une l'autre, se repoussent et ce, en raison d'une politique qui place le peuple du Bas-Canada «dans la dure alternative de se soumettre à un servage ignominieux ou de voir en danger les liens qui l'unissent à la Mère-Patrie» ${ }^{43}$. Par la mise en cuvre d'une politique uniquement déterminée par l'origine, les Britanniques ont donc rendu la condition de sujet britannique intenable, contradictoire, pour les colons français.

D'un autre côté, on essaie de faire de l'allégeance aussi un pôle suffisant pour définir l'identité politique de la colonie. Toutes les

40 Résolution n' 2, Journaux de la Chambre d'Assemblée du Bas-Canada, 1834, 43: 320. Il faut remarquer que cette problématisation est tout à fait conforme à l'approche traditionnelle qui présente l'allégeance comme un pacte conclu entre le roi et ses sujets.

41 Résolution $n^{\circ} 47$, ibid., 321.

42 Résolution $n^{\circ} 53$, ibid., 324.

43 Résolution $n^{\circ} 48$, ibid., 322. 
revendications ou les critiques se fondant sur le postulat que l'Assemblée du Bas-Canada est l'exacte réplique du Parlement de Westminster et qu'elle peut, par conséquent, réclamer les mêmes privilèges, reposent sur un «oubli» de taille: le Bas-Canada n'est pas souverain, il est une colonie au sein d'un empire ${ }^{44}$. Les rapports entre celui-ci et la société métropolitaine ne sont pas ceux qui existent entre les deux versions de la même société, composées d'individus ayant les mêmes droits. Ne pas considérer cela, c'est prétendre fonder l'identité politique en ne considérant que l'allégeance. Ce qui devient paradoxal dans la mesure où l'on postule que le fait d'être sujet de l'empire permet de ne pas tenir compte du caractère britannique, justement, de cet empire.

Il s'agit de nier que le Parlement de Westminster puisse exercer de manière absolue sa souveraineté sur les affaires internes de la colonie, lesquelles seraient du ressort exclusif de l'Assemblée locale. Négation qui se fonde sur l'idée d'égalité naturelle des sujets britanniques et qui implique alors une conception fédéraliste de l'empire britannique ${ }^{45}$. On doit noter ici que la problématique de l'allégeance aurait pu supporter une telle politique. En effet, rappelons que l'allégeance n'est due qu'à la «personne naturelle» du roi. Elle ne crée pas d'obligation envers sa personne politique, c'est-à-dire «le roi en son Parlement». Dans cette optique, conférer un caractère absolu au pôle de l'allégeance peut réellement légitimer la rupture avec l'empire dans la mesure où il s'agit de rompre avec le Parlement anglais. C'est de cette façon que les révolutionnaires américains ont d'abord thématiser leur volonté d'indépendance ${ }^{46}$. Et c'est bel et bien dans cette logique que s'inscrivent les prétentions de l'Assemblée du Bas-Canada de posséder les mêmes privilèges que le parlement anglais, que l'on trouve exprimées dans les résolutions 63 et 79 . Mais le caractère empirique, positif, du pacte social tel que se le représentent les politiciens bas-canadiens implique nécessairement une référence à la

44 On me permettra de rappeler ici l'analyse qu'a fait Henri Brun de ces prétentions: «La constitution de 1791 n'avait pourvu l'Assemblée d'aucun privilège lui permettant de défendre son indépendance. D'autre part, rien ne l'autorisait à se prétendre légataire des coutumes séculaires des Communes anglaises. Jurisprudence et doctrine sont d'une unanimité constante sur ce point.» H. Brun, La formation des institutions parlementaires québécoises (Québec, Presses de l'Université Laval, 1970), 110.

45 On sait qu'une telle conception de l'empire avait cours dans les Treize Colonies avant la guerre d'indépendance. Voir E. Marienstras, Nous le Peuple: les origines du nationalisme américain (Paris, Gallimard, 1988), 163-179.

46 Ces dirigeants coloniaux finissent "par récuser de manière radicale le pouvoir du Parlement sur les colonies. Dans les années 1770, ils considèrent qu'ils ne doivent allégeance qu'à la personne "naturelle" du roi et qu'ils ne font plus partie du même corps politique que les sujets du souverain restés en Angleterre». Élise Marienstras, op. cit., 179. 
personne du roi en son Parlement, à sa personne politique. On rejette alors à la fois l'allégeance à la personne du roi et au Parlement anglais. Et il n'est plus possible, dès lors que la question quitte le terrain de l'allégeance due à la seule personne naturelle du roi, de se réclamer de l'allégeance britannique contre le Parlement. L'allégeance britannique se rompt comme sous le poids d'une surcharge...

C'est un procédé théorique que l'on retrouve encore au centre des résolutions adoptées lors du mouvement des assemblées publiques. L'originalité profonde de ces résolutions, au regard de ce qui nous intéresse ici, est de problématiser l'éclatement de la figure symbolique du sujet britannique en utilisant le thème du droit naturel. L'idée appliquée à la défense des droits des sujets britanniques d'origine française au Bas-Canada ne date pas du mouvement des assemblées publiques. Elle apparaît çà et là bien avant cette date, mais jamais de manière aussi systématique (ce qui s'explique sans doute par le fait que ces assemblées publiques ont été le fruit d'une organisation centralisée, ce qui implique aussi la systématisation du discours politique qui y est tenu). Dans le contexte colonial, le droit naturel vient résoudre le paradoxe que porte une révolte faite au nom de la conservation des droits de sujets britanniques reconnus aux sujets coloniaux de Sa Majesté. Elle désigne en fait un espace juridique antérieur qui permet à la légalité britannique de se rapporter à elle-même, d'être évaluée à l'aide de ses propres critères. Il s'agit là d'une exigence logique. Se met alors en place un dispositif théorique où le droit naturel se présente sous la forme des droits (positifs) des sujets britanniques reconnus aux sujets bas-canadiens. Alors que le contrat social n'est pas ce qui rassemble et organise les individus pour leur assurer la préservation de leurs droits naturels hors de l'état de nature, mais plutôt un traité liant le roi à sa colonie. Forme théorique qui se rapporte sans aucun doute au principe énoncé dans le jugement célèbre de Lord Mansfield:

Les articles d'une capitulation par laquelle s'est opérée la reddition d'un pays et ceux du traité en vertu duquel s'est accomplie la cession sont sacrés et inviolables quant à leur esprit et à leur portée véritable. ${ }^{47}$

La mise en équivalence des droits reconnus aux sujets coloniaux et du droit naturel est le schéma général qui se répète d'une assemblée à l'autre et qui constitue l'essentiel du discours de rupture alors mis en œuvre. Ainsi, l'assemblée du 15 mai à Saint-Laurent s'ouvre-t-elle sur

47 «Jugement de Lord Mansfield dans Campbell vs Hall, 1774», A. Shortt et R. G. Doughty, Documents..., 1: 508. 
l'affirmation que les résolutions Russell «détruisent de fait les droits et les libertés du peuple de cette province, droits et libertés que nous regardons comme les droits de naissance du citoyen anglais solennellement proclamés par l'acte déclaratoire de la $18^{\mathrm{e}} \mathrm{Geo}$ III» ${ }^{48}$. Dans ces conditions, la violation de ces droits par la métropole équivaut à la rupture du contrat social par le souverain, ce qui autorise la rébellion contre le souverain. C'est ce qu'expriment plus que clairement l'ensemble des assemblées ${ }^{49}$.

Par souci d'exactitude, il faudrait mentionner que cette stratégie de légitimation passe parfois par un raisonnement qui correspond plus directement au modèle américain. Ainsi cette résolution qui affirme:

Que depuis un grand nombre d'années, ce pays lutte pour la jouissance de droits et de libertés auxquels ses habitans de toute origine ont droit de prétendre, et par le droit de nature qui les a fait naître égaux à leurs compatriotes d'outre-mer, et par leur qualité de citoyens anglais. ${ }^{50}$

On est alors en présence d'une autre logique, celle qui fonde la possession par les coloniaux des droits de sujets britanniques sur l'égalité naturelle qui les lient avec les sujets métropolitains. Ce qui semble correspondre au processus, déjà analysé, où la figure symbolique du sujet britannique se délite par «absolutisation» du pôle de l'allégeance. Mais il n'est pas indifférent que l'on passe de la médiation par l'idée de contrat social protégeant les droits positifs d'une colonie, à l'affirmation de l'existence de ces droits sur la base d'une égalité naturelle entre tous les hommes, par conséquent entre tous les sujets de Sa Majesté. D'un côté, on aurait la procédure de particularisation telle qu'on l'a décrite précédemment en référence à la pensée de Burke et, de l'autre côté, on aurait une version universalisante, abstraite, du droit de nature. Ce dernier n'est plus le droit (positif) reçu par héritage et protégé par la loi naturelle, mais plutôt le droit (naturel) reçu par tous et chacun en tant que membres de l'espèce humaine. Dans cette optique, ce qui donne la qualité de sujet

48 J.-P. Bemard, dir., Assemblées publiques, résolutions et déclarations de 1837-1838 (Montréal, VLB, 1988), 2.

49 Un exemple: "Que ces actes, ces traités portant des obligations réciproques, savoir de notre part amour et obéissance, de la part de l'Angleterre, protection et garantie de la liberté, seraient virtuellement annulés par la violation de promesse d'une des parties contractantes. ...Que dans ces circonstances, nous ne pourrons regarder autrement le gouvernement qui aurait recours à l'injustice, à la force et à une violation du contrat social, que comme un pouvoir oppresseur, un gouvernement de force, pour lequel la mesure de notre soumission ne devrait être désormais que la mesure de notre force numérique, jointe aux sympathies que nous trouverons ailleurs.» Ibid., 78.

50 «Assemblée de Sainte-Rose (Terrebonne), 11 juin 1837», ibid., 78. 
britannique c'est l'égalité naturelle des hommes au sein de l'empire. Alors que dans le cas précédent, ce qui fonde l'égalité, c'est la qualité de sujet britannique. Cette différence émerge de la crise et de la décomposition de la figure symbolique du sujet britannique. Elle témoigne de ce qu'origine et allégeance se sont déliées et existent dorénavant à l'état libre, chacune pouvant provoquer une autre fondation de l'identité politique.

\section{CONCLUSION}

Il ne s'agit là bien évidemment que d'une hypothèse visant à fournir une nouvelle description (j'insiste sur le terme) de la crise politique telle qu'elle se développe dans la colonie. Cette hypothèse n'apporte rien de nouveau à la connaissance du déroulement des luttes politiques dans la colonie, ni même à l'analyse des motifs qui les supportent. Mais il me semble qu'elle permet de poser le problème au bon niveau d'analyse, soit celui de la crise de l'identité politique. Ainsi échappe-t-on peut-être à l'alternative au sein de laquelle évolue une certaine historiographie: ou bien on sépare les dimensions nationale et sociale de la crise bas-canadienne, ou bien on les rabat une sur l'autre. Ne devrait-on pas plutôt considérer que le politique contient un «moment identitaire», qui n'est pas nécessairement celui de la nation et qui définit la possibilité même de sa crise $^{51}$ ? Ainsi pourra-ton, peut-être, avancer dans l'objectivation de la question.

51 On pourrait, par exemple, suivre les indications fournies par Jean-Louis Vuillierme, Le concept de système politique (Paris, Presses universitaires de France, 1989), 576 p., qui veut établir que le politique loin d'être réductible à l'État, au pouvoir en général ou encore au social, concerne au premier chef ce qui dans la société correspond à la "communauté autonome». Dès lors, le problème de l'identité est partie intégrante de la définition du politique et ne s'y rapporte pas de l'extérieur. 